\title{
Dokumente in zeitlicher Reihenfolge
}





\section{Dokumente in zeitlicher Folge}

Juni/Juli 1919 Berlin Warnung der KPD vor der Errichtung einer Militärdiktatur (Nr. 1)

o. O. Notiz aus den Akten Kapps über die Haltung der Entente zu einem Staatsstreich (Nr. 3)

20./21. 7. 1919 o. O. Aufstandsplan des Garde-Kavallerie-Schützen-Korps (Nr. 2)

1.9.1919 Berlin Forderungen des Reichswehrgruppenkommandos 1 an den Reichswehrminister, das Militär rücksichtslos gegen die revolutionären Kräfte einzusetzen (Nr. 4)

18. 9. 1919 Berlin Denkschrift der Putschisten: „Die Beteiligung von Mehrheitssozialisten an der Regierungsgewalt“ (Nr. 5)

23. 10. 1919 o. O. Stellungnahme zu einem Putschprogramm, in der die Chancen für einen Staatsstreich zum gegenwärtigen Zeitpunkt erwogen werden (Nr.6)

Okt./Nov. 1919 o. O. Exkurs der Putschisten über die „neue“ Reichsverfassung (Nr. 7)

9. 11.1919 Mitau Darlegung der gemeinsamen Interessen der russischen und deutschen Konterrevolutionäre durch den Oberbefehlshaber der Freiwilligen Westarmee in einem Brief an Kapp (Nr. 8)

19.11. 1919 Berlin Oberst Bauer über die Bereitschaft Hindenburgs, sich als Sammelkandidat aller reaktionären Parteien und Verbände bei den kommenden Reichspräsidentenwahlen aufstellen zu lassen (Nr. 9)

30.11.1919 Berlin Aufruf des Vorstandes der SPD an die Arbeiter, in die Einwohnerwehren einzutreten (Nr. 10)

Nov. 1919 Berlin Aufruf der KPD, den Kampf gegen eine drohende Militärdiktatur aufzunehmen (Nr. 11)

10.12. 1919 Berlin Aufruf des Zentralkomitees der USPD zum Kampf gegen die Konterrevolution (Nr. 12)

12.12. 1919 Berlin Aufruf der Zentrale der KPD, die Aufhebung des Belagerungszustandes zur Sammlung der revolutionären Kräfte zu nutzen (Nr. 13)

Dez. 1919 o. O. Aktionsprogramm der Putschisten für den Umsturz (Nr. 14)

o. O. Aufzeichnungen Schnitzlers über geplante Maßnahmen der Putschisten bei Proklamierung des Generalstreiks (Nr. 15) 
XXXIV

8. 1.1920

13. 1.1920

23. 1.1920

Jan. 1920

3. 3.1920

4. 3.1920

8. 3.1920

10. 3. 1920

12. 3.1920

März 1920 1920
Dokumente in zeitlicher Folge

Königsberg Brief der Mitteldeutschen Creditbank, Filiale Königsberg i.Pr., an Geheimrat Kapp über die Bereitschaft verschiedener Banken, seine Bestrebungen zu unterstützen (Nr. 16)

Berlin Gemeinsamer Aufruf der Zentrale der Betriebsräte Deutschlands, der USPD und der KPD, im Kampf gegen das Betriebsrätegesetz die Aktionseinheit der Arbeiterklasse zu schaffen (Nr. 17)

Berlin „Die Rote Fahne“ zur Proklamierung des Ausnahmezustandes (Nr. 18)

Mülheim/Ruhr Hugo Stinnes an den Minister für Wiederaufbau, Otto Geßler, über die Notwendigkeit der Errichtung einer Diktatur (Nr. 19)

Berlin Aufforderung der Zentrale der KPD nach Auflösung der konterrevolutionären Einwohnerwehren und nach Bewaffnung des Proletariats (Nr. 20)

o.O. Erörterung von Möglichkeiten und Voraussetzungen, unter denen ein Putsch erfolgen könnte (Nr. 21)

Mitte Februar

Berlin Erklärung der Zentrale der KPD zu den politischen Hintergründen des Auslieferungsbegehrens (Nr. 22)

Naumburg Bericht an Kapp über die Stimmung der Naumburger Einwohnerwehren und ihre Bereitschaft, den bevorstehenden Staatsstreich zu unterstützen (Nr. 23)

Berlin Verstärkte Machtkonzentration in den Händen des Generals v. Lüttwitz durch die Verordnung des Reichswehrministeriums, Heeresleitung, Allgemeines Truppenamt, Nr. 1900/2.20.T. 2. III (Nr. 24)

Stettin Mitteilung des Direktors des Pommerschen Landbundes an Ludendorff, daß die Geheimhaltung der Putschpläne nicht mehr gewährleistet ist (Nr. 25)

Berlin Warnung der "Roten Fahne“ vor einer drohenden Putschgefahr (Nr. 26)

Berlin Brief Kapps an Ludendorff über die Form der Durchführung des Staatsstreichs (Nr. 27)

Berlin Warnung an die Parteiorganisationen durch die Zentrale der KPD vor überraschenden gewaltsamen Aktionen der militärischen Konterrevolution (Nr. 29)

Berlin Entlarvung der politischen Hintergründe für die Verschiebung der Wahlen zum Reichstag und der Wahl des Reichspräsidenten durch die "Rote Fahne“ (Nr. 31)

Berlin Note der Putschregierung an die deutschen Gesandten und Geschäftsträger im Ausland zur Ubermittlung an die fremden Mächte, um sie zur Tolerierung des Staatsstreichs zu veranlassen (Nr. 30) 
März $1920 \quad$ o. O. Aktionsplan der Putschisten aus den beschlagnahmten Papieren Schnitzlers (Nr. 28)

13. 3. 1920 Berlin Proklamierung des Staatsstreichs durch Kapp und Lüttwitz (Nr. 34)

Berlin „Regierungsprogramm“ der Putschisten (Nr. 35)

Berlin Aufruf der sozialdemokratischen Mitglieder der Reichsregierung und des Parteivorstandes der SPD zum Generalstreik (Nr. 36)

Berlin Aufruf des ADGB und der Afa zum Generalstreik (Nr. 37)

o. O. Stellungnahme des Generalsekretariats der christlichen Gewerkschaften Deutschlands zum Kapp-Putsch (Nr. 38)

Berlin Aufruf der Zentrale der KPD zum Kampf gegen die Militärdiktatur (Nr. 39)

Berlin Stellungnahme der Deutschen Volkspartei zum Putsch (Nr. 40)

Berlin Aufforderung der Reichszentrale für Einwohnerwehren an die Einwohnerwehren zur bewaffneten Unterstützung des Staatsstreichs (Nr. 41)

Chemnitz Aufruf der KPD-Bezirksorganisation ErzgebirgeVogtland zum gemeinsamen Kampf gegen die Putschisten, zur Wahl von Arbeiterräten und zur Bewaffnung des Proletariats (Nr. 121)

Chemnitz Ubernahme von Kontrollfunktionen und Bildung einer Arbeiterwehr durch den Aktionsausschuß Chemnitz (Nr. 122)

Leipzig Appell der Leipziger USPD zum Kampf gegen die Konterrevolution (Nr. 123)

Dresden Ankündigung des Befehlshabers des Wehrkreises IV, jeden Streik rücksichtslos zu unterdrücken (Nr. 124)

o. O. Aufruf der drei Arbeiterparteien des Regierungsbezirkes Merseburg zum Generalstreik (Nr. 167)

Magdeburg Aufforderung des Vollzugsrats Magdeburg zum Generalstreik (Nr. 168)

Stendal Aufruf des Exekutiv-Komitees Stendal zum Kampf gegen die Putschisten (Nr. 169)

Jena Entschließung der revolutionären Betriebsobleute Jenas zum Eintritt in den Generalstreik (Nr. 206)

Altenburg Aufruf der gewerkschaftlichen Organisationen von Sachsen-Altenburg an die Soldaten der Reichswehr zum gemeinsamen Kampf gegen die Putschisten (Nr. 207)

Weimar Aufruf des Staatsrats von Thüringen zur Verteidigung der Verfassung und der Reichsregierung (Nr. 208) 
Weimar Bekenntnis der Reichswehrbrigade 16 zur Putschregierung (Nr. 209)

Cottbus Resolution der Bezirksdelegiertenkonferenz der Bergarbeiter aus der Ober- und Niederlausitz gegen den Kapp-Putsch (Nr. 248)

Breslau Aufruf der sozialdemokratischen „Volkswacht" zum Generalstreik, um die Weimarer Republik gegen die Militärdiktatur zu verteidigen (Nr. 249)

Görlitz Aufruf der SPD und der Gewerkschaften von Görlitz, sich auf den Generalstreik gegen den Staatsstreich vorzubereiten (Nr. 250)

Königsberg Anerkennung der Putschregierung durch den Oberpräsidenten der Provinz Ostpreußen, Winnig, und den Militärbefehlshaber des Wehrkreiskommandos I, Estorff (Nr. 251)

Schwerin Aufruf der Arbeiterparteien und des Gewerkschaftskartells von Schwerin zum Generalstreik (Nr. 282)

Stettin Sympathiekundgebung des Pommerschen Landbundes für das Kapp-Lüttwitz-Regime (Nr. 283)

Stuttgart Appell der KPD-Bezirksorganisation Württemberg, im Kampf gegen die Putschisten nicht die Regierung Ebert-Noske, sondern die Lebensinteressen der Nation zu verteidigen (Nr. 328)

Stuttgart Verurteilung des Putsches durch den SPD-Landesvorstand Württemberg (Nr. 329)

Hof Aufruf des Vollzugsausschusses des Bezirks Hof, die Errungenschaften der Revolution $\mathrm{zu}$ verteidigen und Arbeiterregierungen zu bilden (Nr. 330)

München, Stuttgart, Karlsruhe Gemeinsamer Protest der Regierungen von Bayern, Württemberg und Baden gegen den Staatsstreich (Nr. 331)

München Beratung des bayerischen Ministerrates über die durch den Staatsstreich geschaffene Lage (Nr. 332)

Frankfurt a. $M$. Aufruf der drei Arbeiterparteien Frankfurts zum gemeinsamen Kampf gegen die Militärdiktatur (Nr. 350)

Saarbrücken Solidaritätserklärung der Sozialdemokratischen Partei und der Gewerkschaften des Saargebiets für den Kampf der Arbeiter gegen den Staatsstreich (Nr. 351)

Kassel Sympathieerklärung des Reichswehrgruppenkommandos 2 für die Putschregierung (Nr. 352)

13./14. 3.1920 Spandau Aufruf der drei Arbeiterparteien von Spandau zum Generalstreik (Nr. 42) 
Frankenberg Bekanntmachung des provisorischen Aktionsausschusses Frankenberg über die Bildung einer Arbeiterwehr und die Wahl von Räten in den Betrieben (Nr. 125) Hermsdorf Proklamierung des Generalstreiks durch den Aktionsausschuß Hermsdorf (Nr. 126)

Greiz Aufruf des Aktionskomitees der Betriebsvertrauensleute von Greiz und Umgebung zum Generalstreik (Nr. 210) Mannheim Aufforderung der KPD-Bezirksorganisation $\mathrm{Ba}-$ den, revolutionäre Betriebsräte zu wählen (Nr. 333)

14. 3.1920 Berlin Rundschreiben Nr.40 der Zentrale der KPD an die Bezirksleitungen zum Eintritt in den Generalstreik und zur Herstellung einer gemeinsamen Kampffront gegen die Militärdiktatur (Nr. 43)

Berlin Aufruf der KPD zum gemeinsamen Kampf der Arbeiterklasse gegen die Militärdiktatur und zur Bildung von Arbeiterwehren unter Kontrolle der Arbeiterräte (Nr. 44)

Berlin Aufruf des Zentralkomitees der USPD zum Generalstreik gegen die Militärdiktatur (Nr. 45)

Berlin Aufruf der Berliner Gewerkschaftskommission zum einheitlichen Kampf gegen die Militärdiktatur (Nr. 46)

Brandenburg Aufruf des Aktionsausschusses Brandenburg zum Generalstreik (Nr. 47)

Berlin Entschließung der Beamtenbünde zum Eintritt in die Streikfront, um die Verfassung zu verteidigen (Nr. 48)

o. O. Entwurf eines Beschlusses der Zentral-Arbeitsgemeinschaft Bergbau zur Verhinderung des Generalstreiks und zur Aufrechterhaltung der Arbeitsgemeinschaftspolitik (Nr. 49)

Berlin Aufruf der DDP zur Beteiligung am Generalstreik, um die Verfassung zu verteidigen (Nr. 50)

Berlin Stellungnahme der Deutschnationalen Volkspartei zum Kapp-Putsch (Nr. 51)

Berlin Aufforderung Kapps an die Regierungen der „Bundesstaaten“, Vertreter nach Berlin zu entsenden (Nr. 52)

Berlin Anweisung der Putschregierung an die Militärbefehlshaber, die vollziehende Gewalt zu übernehmen (Nr. 53) Dresden Erklärung der Regierung des Freistaates Sachsen zur Lage (Nr. 127)

Halle Aufruf des Aktionsausschusses für Mitteldeutschland und der Streikleitung Halle zum Kampf gegen die Hochverräter (Nr. 170)

Dessau Aufforderung des Aktionsausschusses Dessau an die Arbeiter, Angestellten und Beamten, sich in die Kampffront gegen die Putschisten einzureihen (Nr. 171) 
Aschersleben Aufruf des Aktionsausschusses Aschersleben zum Generalstreik und zur Aktionseinheit im Kampf gegen die Putschisten (Nr. 172)

Gotha Aufruf des Vollzugsrats Gotha zum gemeinsamen Kampf gegen die Militärdiktatur (Nr. 211)

Mühlhausen/Thür. Aufruf des Vollzugsrats Mühlhausen, im gemeinsamen Kampf die Republik zu verteidigen (Nr. 212) Jena Erklärung der revolutionären Betriebsobleute Jenas, gegen die Militärdiktatur, aber nicht für die Ebert-Bauer zu kämpfen (Nr. 213)

Danzig Aufruf der Kommunistischen Partei des Freistaats Danzig, den Kampf der Arbeiter in Deutschland gegen die Militärdiktatur sowie gegen die Regierung Ebert-Noske durch Eintritt in den Generalstreik zu unterstützen (Nr. 252)

Königsberg Bekenntnis des Kreisvereins Königsberg Stadt der Deutschnationalen Volkspartei zum Staatsstreich (Nr. 253) Forst/Niederlausitz Aufruf des Vollzugsausschusses Forst, im Kampf gegen die Militärdiktatur und das System EbertNoske Betriebsräte zu wählen und Arbeiterwehren zu bilden (Nr. 254)

Senftenberg Aufruf des Aktionsausschusses Senftenberg zum Generalstreik (Nr. 255)

Cottbus Aufruf des Aktionsausschusses Cottbus zum Generalstreik (Nr. 256)

Cottbus Die sozialdemokratische "Märkische Volksstimme“ über Protestdemonstrationen der Cottbuser Arbeiter und ihren Eintritt in den Generalstreik (Nr. 257)

Hirschberg Aufruf des Gewerkschaftskartells Hirschberg zur Bildung einer Arbeiterregierung und zur Bewaffnung des Proletariats (Nr. 258)

Rostock Proklamierung des Generalstreiks durch den Aktionsausschuß Rostock (Nr. 284)

Braunschweig Appell der Arbeiterparteien Braunschweigs zum Generalstreik und zur Herstellung der Aktionseinheit im Kampf gegen die Militärdiktatur (Nr. 285)

Hamburg-Altona Aufforderung Hamburger Gewerkschaftsverbände an die Gewerkschafter, sich in die Kampffront gegen die Putschisten einzureihen (Nr. 286)

Neustrelitz Erklärung des SPD-Parteitages des Landes Mecklenburg-Strelitz zum Kapp-Putsch (Nr. 287)

Schwerin Bekenntnis Lettow-Vorbecks zur Putschregierung und Absetzung der Landesregierung von Mecklenburg-Schwerin (Nr. 288) 
Wilhelmshaven Offene Parteinahme der Marinestation der Nordsee für die Putschregierung (Nr. 289)

Stuttgart Erklärung des Landesverbandes Württemberg der Hirsch-Dunckerschen Gewerkvereine für den gemeinsamen Kampf der Arbeiterklasse gegen die Putschisten (Nr. 334)

Pforzheim Aufruf badischer Gewerkschaften, in einen 24stündigen Generalstreik zu treten (Nr. 335)

o. O. Aufruf der drei Arbeiterparteien des Bezirks Niederrhein zum gemeinsamen Kampf gegen die Putschregierung (Nr. 353)

14./15. 3. 1920 Berlin Aufruf der Eisenbahnergewerkschaften zum Eintritt in den Generalstreik (Nr. 54)

Magdeburg Erklärung der Vertrauensleute der Unteroffiziere der Reichswehrmannschaften von Magdeburg gegen die putschistischen Offiziere (Nr. 173)

Weimar Zustimmung des Thüringer Bauernbundes Weimar zum Staatsstreich (Nr. 214)

15. 3. 1920 Berlin Bericht des Generals Reinhardt über die Vorgänge in Berlin in der Nacht vom 12. zum 13. März 1920 (Nr. 32)

Berlin Aufruf der Zentrale der KPD zur Wahl eines Arbeiterrats für Groß-Berlin (Nr. 55)

Eberswalde Bericht des Extrablatts der revolutionären Arbeiter des Finowtals über den gemeinsamen Kampf der drei Arbeiterparteien gegen die Militärdiktatur (Nr. 56)

Berlin Brief des Vorstandsmitglieds der Badischen Anilin\& Soda-Fabrik Ludwigshafen a/Rhein, Dr. Bueb, an Dr. Oster, Ammoniakwerk Merseburg, Leunawerke Kr. Merseburg, zur Haltung der Chemieindustriellen im Kapp-Putsch (Nr. 57)

Berlin Verordnung des Generals Lüttwitz gegen den Generalstreik (Nr. 58)

Berlin Verordnung Kapps über die Todesstrafe für Streikführer und Streikposten (Nr. 59)

Berlin Bedingungen der Putschisten für Verhandlungen mit der Reichsregierung (Nr.60)

Chemnitz Aufruf der Bezirksleitung der KPD ErzgebirgeVogtland an die Landbevölkerung, sich dem Kampf des Proletariats gegen die Putschisten anzuschließen (Nr. 128)

Plauen (Vogtl.) Aufruf des Aktionsausschusses der drei Arbeiterparteien und des Gewerkschaftskartells Plauen, den Generalstreik geschlossen durchzuführen und eine Arbeiterregierung zu erkämpfen (Nr. 129) 
Zwickau Aufruf des Aktionsausschusses Zwickau, in den Generalstreik gegen die Mliitärdiktatur einzutreten und gemeinsam die Volksrechte zu verteidigen (Nr. 130)

Zwickau Bericht des Rates der Stadt Zwickau an den Amtshauptmann über die Unterstellung der städtischen Behörden unter den Aktionsausschuß (Nr. 131)

Halle Das „Volksblatt“, Halle, zu den Ursachen des KappPutsches und zu den Zielen des Volkskampfes (Nr. 174)

Oberröblingen Erklärung der A. Riebeckschen MontanWerke A.-G. Grubenverwaltung Oberröblingen zwecks Verhinderung des Generalstreiks (Nr. 175)

Magdeburg Bekenntnis des Militärbefehlshabers der Reichswehrbrigade 4 zur Putschregierung (Nr. 176)

Greiz Bericht des Aktionsausschusses der Betriebsvertrauensleute von Greiz und Umgebung über die ersten Aktionen der Greizer Arbeiter gegen den Staatsstreich (Nr. 215)

Weimar Aufruf des Aktionsausschusses Weimar zum Generalstreik (Nr. 216)

Breslau Die sozialdemokratische „Volkswacht" über den Kampf der Breslauer Arbeiter gegen die Putschisten (Nr. 259)

Waldenburg Die „Waldenburger Zeitung“ über die Durchführung des Generalstreiks im Waldenburger Industriegebiet und über die Maßnahmen des Waldenburger Arbeiterrats im Kampf gegen die Militärdiktatur (Nr. 260)

Kattowitz Resolution einer Konferenz der drei Arbeiterparteien in Kattowitz zur Herstellung der Einheitsfront der Arbeiterklasse (Nr. 261)

Danzig „Das freie Volk“ zum Kampf gegen die Konterrevolution in Deutschland und im Freistaat Danzig (Nr. 262)

Stettin Appell des Aktionsausschusses Stettin an die Soldaten der Reichswehr (Nr. 290)

Essen Appell der KPD und USPD von Essen, sich im Kampf gegen die Militärdiktatur um die revolutionären Kräfte zu scharen (Nr. 354)

Hagen Die Hagener „Neue Freie Presse“ über die ersten Kampfmaßnahmen der drei Arbeiterparteien des Bezirks Hagen-Schwelm gegen den Putsch (Nr. 355)

Hamm Bericht über den erfolgreichen Kampf von Bergarbeitern aus Herringen gegen die Einwohnerwehr von Sandbochum (Nr. 356)

Offenbach Das "Offenbacher Abendblatt“ zur Kampfbereitschaft der Massen und zur Forderung nach einer Arbeiterregierung (Nr. 357) 
Solingen Bericht des "Solinger Volksblatts“ über eine Konferenz der drei Arbeiterparteien des Wuppertals, auf der beschlossen wurde, gemeinsam den Kampf gegen die Putschisten aufzunehmen (Nr. 358)

Aachen Aufruf der Aachener „Freien Presse“ zum Kampf gegen Militaristen und Separatisten (Nr. 359)

15./16.3.1920 Wittenberg Die Streikleitung Wittenberg zum Programm der Putschisten (Nr. 177)

Jena Appell des Aktionsausschusses der Freien Sozialistischen Jugend Jenas an die Arbeiterjugend, sich in die Kampffront gegen die Militärdiktatur einzureihen (Nr. 217)

Sorau Beschluß des Ordnungsausschusses Sorau zum Eintritt in den Generalstreik und zur Ubernahme polizeilicher und behördlicher Kontrollfunktionen sowie zur Bildung einer republikanischen Sicherheitswehr (Nr. 263)

Bremen Aufruf der drei Arbeiterparteien Bremens zum gemeinsamen Kampf gegen die Konterrevolution (Nr. 291)

16. 3. 1920 Berlin Aufruf des ZK der USPD zur Fortsetzung des Kampfes gegen die Militärdiktatur und das System Ebert-Noske (Nr. 61)

Stuttgart Erste Aufforderung der Reichsregierung zur Wiederaufnahme der Arbeit (Nr. 62)

Zwickau Der Aktionsausschuß Zwickau über eine Massenkundgebung gegen die Militärdiktatur in Zwickau (Nr. 132)

Zwickau Bericht des Gendarmerie-Inspektors Adler an die Amtshauptmannschaft Zwickau über die in zahlreichen Orten gebildeten Aktionsausschüsse und Arbeiterwehren (Nr. 133) Plauen (Vogtl.) Bericht der sozialdemokratischen „Volksstimme" über die Bildung eines gemeinsamen Aktionsausschusses der drei Arbeiterparteien und der Gewerkschaften zur Durchführung des Generalstreiks in Plauen (Nr. 134)

Limbach Bekanntmachung des Vollzugsrats Limbach, daß die Wahlen zum Arbeiterrat eine kommunistische Majorität ergeben haben (Nr. 135)

Oelsnitz (Vogtl.) Aufruf des Aktionsausschusses Oelsnitz zum gemeinsamen Kampf gegen die Putschisten (Nr. 136)

Halle Aufruf der Arbeiterparteien von Halle, den Generalstreik bis zur restlosen Niederschlagung des Putsches durchzuführen (Nr. 178)

Hettstedt Erklärung des Aktionsausschusses Hettstedt zur Ubernahme von Kontrollfunktionen durch die proletarische Sicherheitswehr (Nr. 179) 
Burg Bekanntmachung des Aktionsausschusses Burg und des Abteilungskommandeurs über die Verhaftung der konterrevolutionären Offiziere der Garnison (Nr. 180)

Stendal Bekanntmachung des Garnisonkommandos Stendal über die Absetzung der konterrevolutionären Offiziere durch die verfassungstreuen Unteroffiziere und Soldaten (Nr. 181) Sonneberg Aufruf des Aktionsausschusses für den Kreis Sonneberg an die Bevölkerung, geschlossen den Kampf gegen die Putschisten aufzunehmen (Nr. 218)

Greiz Forderung des Aktionsausschusses der Betriebsvertrauensleute von Greiz und Umgebung nach Herstellung der Aktionseinheit des Proletariats (Nr. 219)

Gera Bericht des Aktionsausschusses Gera über den Kampf der Geraer Arbeiter gegen die Putschisten (Nr. 220)

Zella-Mehlis Appell gefangener Reichswehrsoldaten an ihre Kameraden, sich auf die Seite der kämpfenden Arbeiter zu stellen (Nr. 221)

Cottbus Die sozialdemokratische „Märkische Volksstimme“ über das Blutbad in Cottbus am 15. März 1920 (Nr. 264)

Hannover Auszug aus einer Erklärung des Aktionsausschusses Hannover über die Verurteilung des Kappschen Staatsstreichs und des partikularistischen Welfenputsches (Nr. 292)

Hamburg Aufforderung der USPD-Bezirksorganisation Wasserkante zum Kampf gegen die Militärdiktatur und das EbertNoske-System (Nr. 293)

Hamburg Die Hamburger "Rote Fahne" über die Errichtung der Militärdiktatur in Kiel (Nr. 294)

Harburg Das sozialdemokratische Harburger „Volksblatt" zum Sieg der Harburger Arbeiter über die „Eiserne Schar" des Hauptmann Berthold (Nr. 295)

Stuttgart Aufforderung der KPD-Bezirksorganisation Württemberg an die Arbeiter, sich zu bewaffnen und Arbeiterräte zu bilden (Nr. 336)

München Aufforderung des Aktionsausschusses der Betriebsräte Münchens, den Kampf gegen die Putschisten aufzunehmen (Nr. 337)

Pforzheim Aufforderung der Vorsitzenden der KPD und USPD Pforzheims an die SPD-Führung, sich dem gemeinsamen Kampf der Arbeiterklasse anzuschließen (Nr. 338)

Bayreuth Proklamierung eines 24stündigen Generalstreiks durch den Aktionsausschuß Bayreuth (Nr. 339) 
Heidelberg Forderungen der Heidelberger Werktätigen nach Verstaatlichung der Schwerindustrie und des Großgrundbesitzes (Nr. 340)

Würzburg Bericht des „Fränkischen Volksfreunds“ über den Sturz der sozialdemokratischen Regierung Hoffmann (Nr. 341) München Meldung des WTB über die Drohung bayerischer Bauernbünde mit einem "Gegenstreik“ (Nr. 342)

Hagen Bericht des Landrats des Landkreises Hagen an den Regierungspräsidenten in Arnsberg über die Kämpfe bei Wetter und Herdecke (Nr. 360)

Herdecke Stellungnahme der Vertrauensleute des zweiten Bataillons vom Freikorps Lichtschlag gegen ihre Offiziere (Nr. 361)

16./17. 3.1920 Chemnitz Aufruf der Bezirksleitung der KPD Erzgebirge/ Vogtland an das revolutionäre Proletariat (Nr.137)

Greifswald Appell des Aktionsausschusses Greifswald an die Arbeiter und Soldaten zum gemeinsamen Kampf gegen die Konterrevolution (Nr. 296)

17. 3. 1920 Berlin Sitzungsprotokoll des geschäftsführenden Vorstandes der Zentralarbeitsgemeinschaft über den Rücktritt der Putschregierung und den Abbruch des Generalstreiks (Nr.63)

Berlin Bekanntgabe des Rücktritts Kapps (Nr.64)

Potsdam Aufruf der Reichswehrbrigade 3 zur Niederschlagung der revolutionären Arbeiterbewegung (Nr.65)

Berlin Bekanntgabe des Rücktritts Lüttwitz' und der Ubernahme seiner Funktion durch Seeckt (Nr. 66)

Berlin Aufforderung des Vizekanzlers Schiffer zum Abbruch des Generalstreiks (Nr.67)

Stuttgart Aufruf der sozialdemokratischen Fraktion der Nationalversammlung zum Abbruch des Generalstreiks (Nr. 68) Chemnitz Auszug aus dem Bericht des „Kämpfer" über die Bildung von Dezernaten und die Kontrolle der Banken durch den Vollzugsrat Chemnitz (Nr. 138)

Auerbach Bericht des Aktionsausschusses Auerbach über erfolgreiche Kämpfe der Arbeiterwehr gegen Reichswehrtruppen (Nr. 139)

Frankenberg Aufruf des Vollzugsausschusses Frankenberg an die werktätigen Bauern von Frankenberg und Umgebung, den Kampf der Arbeiterklasse durch Ablieferung von Lebensmitteln zu unterstützen sowie Landarbeiter- und Kleinbauernräte zu bilden (Nr. 140)

Dresden Versuch der sächsischen Regierung, die Aktionsausschüsse zur Zusammenarbeit mit den Behörden zu veranlassen, um sie dann entmachten zu können (Nr.141) 
Staßfurt Bekanntmachung des Magistrats und des Vollzugsrats Staßfurt über die Maßnahmen gegen die konterrevolutionären Truppen und die Bildung einer Arbeiterwehr (Nr. 182) Zschornewitz Bericht der Streikleitung Zschornewitz über den Generalstreik der Zschornewitzer Kraftwerker (Nr. 183) Zeitz Forderungen der Zeitzer Arbeiterschaft für den Abbruch des Generalstreiks (Nr. 184)

Greiz Aufforderung des Aktionsausschusses der Betriebsvertrauensleute von Greiz und Umgebung, für wahrhaft demokratische Verhältnisse zu kämpfen (Nr. 222)

Gera Appell des Aktionsausschusses Gera an die Arbeiter, durch Eintritt in die Rote Armee den Sieg über die Putschisten zu sichern (Nr. 223)

Hoyerswerda Das „Hoyerswerdaer Kreisblatt“ über den Generalstreik in der Stadt (Nr. 265)

Waldenburg Bericht der "Schlesischen Bergwacht" über die Kampfbereitschaft der Massen im Waldenburger Gebiet (Nr. 266)

Wismar Aufruf der KPD-Bezirksorganisation Mecklenburg, gegen die Rückkehr der Ebert-Noske zu kämpfen und revolutionäre Räte zu wählen (Nr. 297)

Stettin Appell des Aktionsausschusses Stettin an die Soldaten zur Solidarität mit den Arbeitern im Kampf gegen die Feinde des Volkes (Nr. 298)

Stettin Forderungen des Aktionsausschusses Stettin nach Entwaffnung der konterrevolutionären Verbände und Bewaffnung des Proletariats (Nr. 299)

München Niederschrift über die zwischen dem bayerischen Staatsministerium und der Streikleitung München über den Abbruch des Generalstreikes geführten Verhandlungen (Nr. 343)

Göppingen Das „Mitteilungsblatt des Aktionsausschusses Göppingen" über die örtlichen Aktionen gegen den KappPutsch (Nr. 344)

Reutlingen Bericht der sozialdemokratischen „Freien Presse“ über die Bildung von Aktionsausschüssen und die Durchführung des Generalstreiks in mehreren württembergischen Städten (Nr. 345)

Dortmund Meldung über die Eroberung Dortmunds durch die bewaffneten Arbeiter (Nr. 362)

Hagen Aufruf der Hagener Zentrale zum Ausbau der Arbeiterwehr (Nr. 363)

Berlin Auszug aus einer Instruktion des Unterstaatssekretärs Haniel an den deutschen Geschäftsträger in Paris, die 
Zustimmung der Alliierten zur militärischen Niederschlagung des Ruhrproletariats zu erwirken (Nr. 364)

17./18. 3. 1920 Berlin Aufruf Seeckts und Schiffers zum Kampf gegen die revolutionäre Arbeiterbewegung (Nr.69)

18. 3.1920 Berlin Aufruf der Zentrale der KPD zur Fortsetzung des Generalstreiks und zur Bewaffnung der Arbeiterklasse (Nr. 70)

Berlin Appell der Zentrale der KPD an die Reichswehrangehörigen (Nr. 71)

Berlin Neunpunkteprogramm des Allgemeinen Deutschen Gewerkschaftsbundes, der Arbeitsgemeinschaft freier Angestelltenverbände und des Deutschen Beamtenbundes (Nr. 72) Berlin Aufruf des Allgemeinen Deutschen Gewerkschaftsbundes, der Arbeitsgemeinschaft freier Angestelltenverbände und des Deutschen Beamtenbundes zur Fortsetzung des Generalstreiks bis zur Erfüllung der gewerkschaftlichen Forderungen (Nr. 73)

Berlin Anweisung des Reichswehrgruppenkommandos 1 an die unterstellten Reichswehrkommandos zum rücksichtslosen Kampf gegen die revolutionären und die anderen demokratischen Kräfte (Nr. 74)

Mittweida Protest des Aktionsausschusses Mittweida an das sächsische Innenministerium gegen die beabsichtigte Entmachtung der Aktionsausschüsse (Nr. 142)

Chemnitz Forderungen der Arbeiterräte Sachsens nach Entwaffnung der konterrevolutionären Verbände und Schaffung einer Arbeiterwehr sowie Wahl revolutionärer Betriebsräte (Nr. 143)

Hohenstein-Ernstthal Bericht des „Hohenstein-Ernstthaler Anzeigers" über die Entwaffnung der Glauchauer Einwohnerwehr durch revolutionäre Arbeiter (Nr. 144)

Eisleben Bericht des Aktionsausschusses für Eisleben und die beiden Mansfelder Kreise über den Kampf der Arbeiter gegen einen Panzerzug der Reichswehr (Nr. 185)

Eisleben Der Aktionsausschuß für Eisleben und die beiden Mansfelder Kreise über die Erfolge des Proletariats im Kampf gegen die Putschisten (Nr. 186)

Eisleben Bekanntmachung des Aktionsausschusses für Eisleben und die beiden Mansfelder Kreise zur Ubernahme polizeilicher Funktionen durch den Aktionsausschuß (Nr. 187)

Bernburg Bericht der sozialdemokratischen Bernburger "Volkswacht" über die Entwaffnung der Zeitfreiwilligen und die Absetzung reaktionärer Offiziere (Nr. 188) 
Merseburg Aufruf des Aktionsausschusses Merseburg, den Kampf solange weiterzuführen, bis die Forderungen der Arbeiter durchgesetzt sind (Nr. 189)

Königsberg Bekanntgabe der von USPD, SPD und freien Gewerkschaften in Ostpreußen gestellten Forderungen an die Reichsregierung nach Zurückdrängung der Konterrevolution, Durchsetzung der Interessen der Werktätigen und Herstellung guter Beziehungen zu Sowjetrußland (Nr. 267)

Senftenberg Anweisung des Niederlausitzer Bergbauvereins an die Bergwerksdirektoren, Repressalien gegen streikende Arbeiter und Angestellte zu ergreifen (Nr. 268)

Wismar Antrag der „Freiwilligen Sturm-Abteilung“ Roßbach an die Reichswehrbrigade 9, verhaftete Teilnehmer einer Versammlung des Deutschen Landarbeiter-Verbandes wegen Aufforderung zum Generalstreik und Waffenbesitz zu erschießen (Nr. 300)

Nürnberg Aufruf der KPD-Ortsgruppe Nürnberg, den Generalstreik zu verschärfen und sich zu bewaffnen (Nr. 346)

Elberfeld Bekanntmachung von Oberbürgermeister und Wehrausschuß in Elberfeld zur Ubernahme des Sicherheitsdienstes durch die Arbeiterwehr (Nr. 365)

Düsseldorf Beschluß der Arbeitsgemeinschaft der rheinischwestfälischen Eisen- und Stahlindustrie zur Durchbrechung der einheitlichen Streikfront der Ruhrarbeiter (Nr. 366)

18./19.3.1920 Berlin Verhandlungen zwischen Regierungsmitgliedern, Koalitionsparteien und Freien Gewerkschaften über das Neunpunkteprogramm (Nr. 75)

Erfurt Aufruf des rechtssozialdemokratischen Zivilkommissars Apel zum Abbruch des Generalstreiks (Nr. 224)

Altenburg Forderungen der SPD Ostthüringens und der SPDLandtagsfraktion von Sachsen-Altenburg (Nr. 225)

17.-19. 3. 1920 Rostock Aufzeichnungen und Anordnungen des Oberbefehlshabers der Rostocker Arbeiterwehr (Nr. 301)

19. 3. 1920 Berlin (Stuttgart) Erneuter Aufruf der Reichsregierung zum Abbruch des Generalstreiks (Nr. 76)

Berlin Aufruf der Zentrale der KPD an die Betriebsangehörigen der Eisenbahn- und Postverwaltungen, den Streik bis zur bedingungslosen Kapitulation der Militaristen fortzusetzen (Nr. 77)

Berlin Appell der Zentrale der KPD an die Soldaten der Reichswehr, sich nicht gegen die Arbeiter mißbrauchen zu lassen (Nr. 78)

Berlin Losungen der Zentrale der Freien Sozialistischen Jugend des Bezirkes Groß-Berlin (Nr. 79) 
Berlin Aufruf des ZK der USPD zur Fortsetzung des Kampfes gegen die Konterrevolution (Nr. 80)

Berlin Aufruf der Berliner Zentralstreikleitung zur entschlossenen Fortführung des Generalstreiks und zur Aufrechterhaltung der Aktionseinheit (Nr. 81)

Berlin Forderung der Berliner Gewerkschaftskommission nach Errichtung einer Arbeiterregierung und Schaffung einer Arbeiterwehr (Nr. 82)

Leipzig Aufruf des Gewerkschaftskartells, der USPD und der SPD von Leipzig, den Kampf abzubrechen und die Arbeit wieder aufzunehmen (Nr. 145)

Meißen Bericht der sozialdemokratischen „Volkszeitung“, Meißen, über den Kampf der Arbeiter im Bezirk Pirna gegen den Kapp-Putsch (Nr. 146)

Magdeburg Aufruf des Vorstands des Sozialdemokratischen Vereins Magdeburg zum Abbruch des Generalstreiks (Nr. 190) Halle Aufforderung des Garnisonältesten von Halle zum Kampf gegen die revolutionäre Arbeiterbewegung (Nr. 191) Gotha Aufruf des Aktionsausschusses Gotha, den Generalstreik und den bewaffneten Kampf fortzusetzen (Nr. 226)

Jena Bericht der "Neuen Zeitung“ über den Entschluß der USPD-Mitglieder in Jena, den Kampf gegen die Konterrevolution fortzusetzen und eine Arbeiterregierung $\mathrm{zu}$ bilden (Nr. 227)

Kassel Gemeinsamer Erlaß des Befehlshabers der Reichswehrbrigade 11 und Regierungsvertretern zur Einleitung des Rachefeldzuges gegen das Proletariat in Thüringen (Nr. 228) Hoyerswerda Forderung der SPD Hoyerswerda an den Reichspräsidenten Ebert nach Bestrafung der Putschisten, Auflösung der konterrevolutionären Verbände, Bewaffnung der Arbeiter und Umbildung der Regierung (Nr. 269)

Frankfurt a. O. Zusammenfassender Bericht des sozialdemokratischen „Volksfreunds" über den Kampf der Frankfurter Arbeiter gegen den Kapp-Putsch (Nr. 270)

Königsberg Aufruf des Ostdeutschen Heimatdienstes zur Bildung einer Kampffront gegen die revolutionäre Arbeiterklasse (Nr. 271)

Stettin Aufforderung des Aktionsausschusses Stettin, den Kampf bis zur endgültigen Niederschlagung der Konterrevolution fortzusetzen (Nr. 302)

Hamburg Forderung einer USPD-Versammlung in Hamburg, erneut in den Generalstreik zu treten, um die Entwaffnung der Arbeiter zu verhindern (Nr. 303) 
Hagen Aufruf des Aktionsausschusses Hagen, den Kampf gegen die Konterrevolution fortzusetzen und die Arbeiterwehren zu erhalten (Nr. 367)

Solingen Das "Ruhr-Echo" zum Sieg der Roten Armee in Remscheid (Nr. 368)

20. 3. 1920 Berlin Vereinbarungen zwischen den Freien Gewerkschaften einerseits und den Vertretern der preußischen Regierung und den Koalitionsparteien im Reich und in Preußen andererseits über das Neunpunkteprogramm der Gewerkschaften (Nr. 83) Berlin Beschluß der Freien Gewerkschaften zum Abbruch des Generalstreiks (Nr. 84)

Berlin Zustimmung des Ausschusses der Gewerkschaftskommission Berlins und Umgebung zum Streikabbruch (Nr. 85) Berlin Aufruf der Zentrale der KPD, dem Beschluß der Zentralstreikleitung zur Fortsetzung des Generalstreiks Folge zu leisten (Nr. 86)

Berlin Die Zentrale der KPD über das Ziel der Partei im gegenwärtigen Zeitpunkt (Nr. 87)

Plauen (Vogtl.) Aufforderung des Vollzugsausschusses Plauen an die Arbeiter, den Generalstreik zu beenden (Nr. 147)

Dessau Bericht des „Anhalter Anzeigers“, Dessau, über die bewaffneten Kämpfe bei Jeßnitz (Nr. 192)

Halberstadt Auszug aus einer Erklärung des sozialdemokratischen „Halberstädter Tageblatts“, in der die Entfernung aller Reaktionäre aus der Reichswehr und dem Verwaltungsapparat gefordert wird (Nr. 193)

Ohrdruf Aufruf des Soldatenrats der 1. Volkswehrarmee an die Arbeiter zum Eintritt in die Volkswehr (Nr. 2209)

Ohrdruf Orientierung der Volkswehr auf die militärischen und ökonomischen Aufgaben im gegenwärtigen Kampf der Thüringer Arbeiter (Nr. 230)

Erfurt Aufruf des Vollzugsausschusses der Arbeiter- und Angestellten-Ausschüsse Erfurts zur Weiterführung des Generalstreiks (Nr. 231)

Jena Bericht der „Neuen Zeitung“ (Jena) über die Bereitschaft der Massen zur Fortsetzung des Generalstreiks (Nr. 232)

Waldenburg Ubernahme der Kontrolle der Behörden im Kreis durch den Arbeiterrat von Waldenburg (Nr. 272)

Schwerin Vereinbarung zwischen dem Staatsministerium von Mecklenburg-Schwerin, Vertretern von Aktionsausschüssen des Landes und dem neuen Militärbefehlshaber über den Abbruch des Generalstreiks (Nr. 304) 
Essen Das „Ruhr-Echo“ über die Befreiung Essens durch die Rote Armee (Nr. 369)

Duisburg Proklamierung der "Diktatur des Proletariat" durch den provisorischen Vollzugsrat von Duisburg (Nr. 370)

21. 3.1920

Potsdam Aufruf des Aktionsausschusses Potsdam an die Soldaten zum gemeinsamen Kampf (Nr. 88)

Cottbus Auszug aus der sozialdemokratischen „Märkischen Volksstimme“ über die Kämpfe der Niederlausitzer Arbeiterwehren gegen die Truppen Buchruckers (Nr. 273)

Pasewalk Forderung des Aktionsausschusses Pasewalk nach Entfernung der putschistischen Offiziere (Nr. 305)

Stettin Der Stettiner „Kämpfer" über Massenproteste und Verteidigungsmaßnahmen der Stettiner Arbeiter gegen das Ultimatum der Militaristen und rechten sozialdemokratischen Führer (Nr. 306)

Hagen Aufruf des Aktionsausschusses Hagen zur Bildung einer provisorischen militärischen und politischen Zentrale (Nr. 371)

Hagen Aufruf der drei Arbeiterparteien von Hagen zur Organisierung der Arbeiterwehr im Kampf gegen die Konterrevolution (Nr. 372)

Hagen Forderung der drei Arbeiterparteien des Industriegebiets an Reichspräsident Ebert, den Vormarsch der konterrevolutionären Truppen einzustellen (Nr. 373)

Angermund Bekanntmachung des Vollzugsrats Angermund zur Ubernahme behördlicher, polizeilicher und militärischer Funktionen (Nr. 374)

Berlin Festlegung der Taktik gegen das siegreiche Ruhrproletariat durch das Reichsministerium und das Preußische Staatsministerium (Nr. 375)

21./22. 3.1920 Leipzig Einschätzung der Ergebnisse des Kampfes der Arbeiter gegen die Putschisten durch die KPD-Ortsgruppe Leipzig (Nr. 148)

22.3.1920 Berlin Rundschreiben Nr. 42 der Zentrale der KPD an die Bezirksleitungen über den Kampf gegen den Kapp-Putsch und die weiteren Aufgaben der Partei nach dem Abbruch des Generalstreiks (Nr. 89)

Berlin Aufzeichnung einer Besprechung bei Reichskanzler Bauer zur Bildung einer Arbeiterregierung (Nr. 90)

Berlin Beschluß des ADGB, der Afa, der Berliner Gewerkschaftskommission, der USPD und der SPD über die Beendigung des Generalstreiks (Nr. 91) 
Glauchau Anordnung des Vollzugsrats Glauchau, Waffen und Munition im Bereich der Amtshauptmannschaft Glauchau an die Aktionsausschüsse abzuliefern (Nr. 149)

Frankenberg Aufruf der Wehrkommission des Frankenberger Arbeiterrats zur Aufrechterhaltung des bewaffneten Wachdienstes (Nr. 150)

Schönebeck Bericht der "Schönebecker Zeitung“ über den Protest einer Volksversammlung gegen die Einsetzung Seeckts und den Entschluß zur Fortführung des Generalstreiks (Nr. 194)

Gotha Zusammenfassender Bericht des Vollzugsrats Gotha über den Kampf der Gothaer Arbeiter gegen den Kapp-Putsch (Nr. 233)

Erfurt Appell des Vollzugs-Ausschusses Erfurt an die Arbeiterschaft, den Kampf bis zur Erfüllung der vom Vollzugsausschuß gestellten Mindestforderungen weiterzuführen (Nr. 234) Breslau Bericht der "Volkswacht" über eine von der KPD einberufene Volksversammlung in Breslau am 21. März 1920 (Nr. 274)

Stettin Der Stettiner „Kämpfer“ über die Forderungen der Landarbeiter Pommerns nach umfassender Verbesserung ihrer wirtschaftlichen, sozialen und rechtlichen Lage (Nr. 307) Kiel Bericht der „Hamburger Volks-Zeitung“ über den Kampf der Kieler Arbeiter gegen die Putschisten (Nr. 308) Regensburg Bericht der sozialdemokratischen „Neuen Donau Post“ über die Durchführung des Generalstreiks und einen bürgerlichen Gegenstreik in Regensburg (Nr. 347)

Essen Bericht des „Ruhr-Echo“ über den weiteren Vormarsch der Roten Armee (Nr. 376)

Köln Die „Rheinische Zeitung“ über Stärke und Organisation der Roten Ruhrarmee (Nr. 377)

22./23. 3. 1920 Spandau Bericht eines republikanischen Reserveleutnants über den Uberfall von Baltikumern auf Spandau-Hennigsdorf (Nr. 92)

Leipzig Einschätzung der Vorgänge in Leipzig durch die Bezirksleitung der KPD (Nr. 151)

o. O. Bericht über die sozialpolitischen Forderungen der außerordentlichen Generalversammlung der Ortsgruppe Dalberg des Deutschen Landarbeiter-Verbandes am 21. März 1920 (Nr. 309)

Marl Eidesformel der Roten Armee (Nr. 378)

23. 3.1920 Berlin Stellungnahme der Zentrale der KPD zur Bildung einer Arbeiterregierung (Nr. 93) 
Berlin Die „Freiheit“ über die Ermordung von Arbeitern in Köpenick (Nr. 94)

Gera Bericht der „Reußischen Tribüne“, Gera, über die Kämpfe der Arbeiter gegen konterrevolutionäre Reichswehrtruppen bei Zickra (Nr. 235)

Allstedt Forderungen des Aktions-Ausschusses für den Amtsbezirk Allstedt zur Zurückdrängung der Konterrevolution und nach Bildung einer Arbeiterregierung (Nr. 236)

23./24. 3. 1920 Magdeburg Resolution der Versammlung des Bezirkes Süd des Sozialdemokratischen Vereins Magdeburg gegen die bisherige Koalitionspolitik (Nr. 195)

Stralsund Forderungen des SPD-Unterbezirks Vorpommern (Nr. 310)

24. 3.1920 Fürstenwalde Zusammenfassender Bericht der „Fürstenwalder Zeitung“" über den Kampf der Fürstenwalder Arbeiter gegen den Kapp-Putsch (Nr. 95)

Chemnitz Der „Kämpfer“ über das veränderte Kräfteverhältnis nach dem Putsch (Nr.96)

Berlin Erklärung des Zentralkomitees der USPD zu den Aufgaben einer Arbeiterregierung (Nr. 97)

Dresden Auszug aus einem Bericht des Amtshauptmanns von Stollberg an das sächsische Ministerium des Innern über die Landesversammlung der Arbeiterräte in Chemnitz (Nr. 152)

Königsberg Verordnung des Wehrkreiskommandos I über die Inhaftierung von Kommunisten und die Bekämpfung von Arbeiterwehren (Nr. 275)

o. O. Anweisungen der Gefechtsleitung Kuhn an die unterstellten Kompanieführer der Roten Armee (Nr. 381)

Bielefeld Vereinbarungen über den Abbruch der Kämpfe im Ruhrgebiet (Bielefelder Abkommen) (Nr. 382)

25. 3. 1920 Berlin Aufruf der Zentrale der Betriebsräte an das werktätige Volk, den Erfolg des Generalstreiks durch Bewaffnung des Proletariats und Bildung von Arbeiterwehren zu sichern (Nr. 98)

Berlin Sozialdemokratische Stellungnahme zur Bildung einer Arbeiterregierung (Nr. 99)

Sebnitz Weigerung des Arbeiterrates Sebnitz, die Waffen der Einwohnerwehr an den Stadtrat abzuliefern (Nr. 153)

Frankenberg Forderung der Frankenberger Arbeiterwehr, die lokalen Wehren zu größeren Verbänden zu vereinigen (Nr. 154) 
Waldenburg Die "Schlesische Bergwacht" über gemeinsame Entwaffnungsaktionen von Arbeitern und Soldaten in Breslau (Nr. 276)

Waldenburg Die "Schlesische Bergwacht" zum Uberfall des Freikorps Faupel auf Hirschberg (Nr. 277)

Neubukow Auszug aus dem Bericht der Station Neubukow des 4. Beritts der Mecklenburg-schwerinschen Landesgendarmerie an das Distriktamt des 4. Beritts in Bützow zum Generalstreik der Landarbeiter und zur Beschlagnahme der Waffen auf dem Lande (Nr. 311)

Münster Meldungen über den Bruch des Bielefelder Abkommens durch die Reichswehr und über Bestrebungen rechter USPD-Führer zur Isolierung der KPD (Nr. 383)

Barmen Die „Freie Presse“ über die Kämpfe in Barmen (Nr. 384)

26. 3. 1920 Berlin Aufstellung von militärischen, politischen und wirtschaftlichen Forderungen durch den Aktionsausschuß der Vollversammlung der Betriebsräte Berlins (Nr. 100)

Berlin Aufruf des ZK der USPD zur Wahl von Betriebsräten (Nr. 101)

Plauen Die „Volkszeitung für das Vogtland“ über die Notwendigkeit, eine Arbeiterregierung zu bilden (Nr. 155)

Halle Forderung der Sozialistischen Studentengruppe Halle an den preußischen Kultusminister, die Universität vom reaktionären Geist zu reinigen (Nr. 196)

Gotha Protest des "Gothaer Volksblatts" gegen den weißen Terror in Gotha und Bad Thal (Nr. 237)

Rostock Die Rostocker „Volkswacht" zum Uberfall der Baltikumer auf Gnoien (Nr. 312)

Essen Das „Ruhr-Echo“ zu den Auseinandersetzungen über das Bielefelder Abkommen auf der Konferenz der Vollzugsräte des Ruhrgebiets in Essen am 25. März 1920 (Nr. 385)

Hagen/Westf. Der Vorsitzende der Hagener Zentrale, Josef Ernst, an Reichskommissar Severing über die Einstellung des Kampfes in Mülheim (Nr. 386)

27.3. 1920 Berlin „Die Rote Fahne“ zur Herstellung der Einheitsfront im Kampf um eine Arbeiterregierung (Nr. 102)

Eisleben Bericht der "Mansfelder Volks-Zeitung“ über den Kampf der Helbraer Arbeiter gegen den Kapp-Putsch (Nr. 197)

Merseburg Auszug aus dem DDP-Organ „Merseburger Korrespondent" über den Kampf der Arbeiter gegen den KappPutsch in Merseburg und Umgebung (Nr. 198) 
Breslau Die sozialdemokratische „Volkswacht“ über den gemeinsamen Kampf von Arbeitern und Soldaten gegen die konterrevolutionären Offiziere in Glatz (Nr. 278)

Hagen Aufruf des Zentralrats an die Kampfleiter, die Kämpfe einzustellen und von der Regierung die Erfüllung des Bielefelder Abkommens zu verlangen (Nr. 387)

Essen Anordnung des Zentralrats der Vollzugsräte für das Industriegebiet Rheinland-Westfalen zur Löhnung und Verpflegung der Roten Armee (Nr. 388)

Gelsenkirchen Nichtanerkennung des Bielefelder Abkommens durch den Provokateur Gottfried Karusseit (Nr. 389)

Berlin „Die Rote Fahne“ über die Notwendigkeit, die errungenen Machtpositionen im Ruhrgebiet zu sichern und die Truppentransporte zu verhindern (Nr. 390)

27./28. 3.1920 Rostock Zusammenfassender Bericht der Rostocker „Volkswacht" über den Kampf der Schweriner Arbeiter gegen den Kapp-Putsch (Nr. 313)

28. 3. 1920 Berlin Aufruf der Zentrale der KPD, die militärische Niederschlagung des Proletariats zu verhindern (Nr. 103)

Dresden Bericht des Wehrkreiskommandos IV an das Reichswehrgruppenkommando 2, Kassel, daß gegenwärtig in Ostthüringen militärische Operationen unmöglich sind und die Entwaffnung der Arbeiter nur auf dem Verhandlungswege erreicht werden kann (Nr. 238)

Rostock Bericht der sozialdemokratischen „Mecklenburgischen Volks-Zeitung“ über den Generalstreik in Bützow und Umgebung sowie den gemeinsamen Kampf von Industrieund Landarbeitern gegen die Rostocker Zeitfreiwilligen bei Katelbogen (Nr. 314)

Rostock Protokoll über die Ermordung des Vertrauensmannes des Landarbeiterverbandes Paul Brümmer (Nr. 315)

Essen Resolution des Zentralrats und der Vollversammlung der Vollzugsräte Rheinland-Westfalens über die Zerschlagung des Militarismus als unmittelbares Ziel des Kampfes des Ruhrproletariats (Nr. 391)

Berlin Ultimatum der Reichsregierung an das Ruhrproletariat (Nr. 392)

29. 3.1920 Berlin Die „Freiheit“ über die öffentlichen Versammlungen der USPD-Bezirksorganisation Berlin-Brandenburg am 28. März 1920 zur Bildung einer Arbeiterregierung (Nr. 104)

Berlin Erlaß des preußischen Innenministers zur Entmachtung der Vollzugs- und Arbeiterräte (Nr. 105) 
Schwarzenberg Auszug aus dem Bericht der Amtshauptmannschaft Schwarzenberg an das sächsische Ministerium des Innern über die Waffenbeschlagnahme in Schwarzenberg auf Initiative Ernst Schnellers (Nr. 156)

Waldenburg Forderung des Arbeiterrats des niederschlesischen Industriegebiets, eine Arbeiterregierung zu bilden und eine Arbeiterwehr einzurichten (Nr. 279)

Stavenhagen Bericht der Kreisbehörde für Volksernährung Stavenhagen des Kommunalverbandes Malchin an das Ministerium des Innern in Schwerin über die Ermordung des Arbeiterfunktionärs Seidel (Nr. 316)

Essen Annahme des Ultimatums der Reichsregierung durch den Zentralrat (Nr. 393)

Münster Unerfüllbare Zusätze des Befehlshabers des Wehrkreises VI zu dem Ultimatum der Reichsregierung vom 28. März 1920 (Nr. 394)

Essen Proklamierung des erneuten Generalstreiks durch den Zentralrat der Vollzugsräte (Nr. 395)

29./30. 3. 1920 Berlin Aufruf der KPD zur Aktionseinheit der Arbeiterklasse und zur Bildung von Arbeiterwehren im Kampf gegen die Konterrevolution und den weißen Terror (Nr. 106)

Rostock Aufforderung des USPD-Bezirksvorstandes Mecklenburg an das Proletariat, den Kampf zur Bildung von Arbeiterregierungen im Reich und in Mecklenburg fortzusetzen (Nr. 317)

30.3.1920 Berlin Auszug aus der Entschließung der Delegiertenvollversammlung der Betriebsräte zum erneuten Generalstreik, um das Ruhrproletariat zu retten und die Bewaffnung der Arbeiterklasse durchzusetzen (Nr. 107)

Glauchau Forderungen des Vollzugsrats Glauchau zur Weiterführung des Volkskampfes (Nr. 157)

Oelsnitz (Vogtl.) Bekanntgabe der Neubildung des Arbeiterrats Oelsnitz und der Ubernahme der Kontrolle über die Stadtverwaltung (Nr. 158)

Altenburg Forderung des Staatsministeriums von SachsenAltenburg an den Reichspräsidenten, die verfassungstreuen Unteroffiziere und Mannschaften der Garnison Altenburg vor Racheakten reaktionärer Militärs zu schützen (Nr. 239)

Gotha Befehl der Brigade Rumschöttel zur Auflösung der Aktionsausschüsse und Entwaffnung der Arbeiter in Thüringen (Nr. 240)

Stettin Protest des Stettiner „Kämpfer" gegen die Truppentransporte nach dem Ruhrgebiet (Nr. 318) 
Berlin Appell der Zentrale der KPD, das Ruhrproletariat vor dem Rachefeldzug der Militaristen zu schützen und damit zugleich die deutsche Arbeiterklasse gegen die Angriffe der Konterrevolution zu verteidigen (Nr. 396)

Berlin Aufforderung der „Roten Fahne“, beim Uberfall der Reichswehr auf das Ruhrgebiet erneut in den Generalstreik zu treten (Nr. 397)

31.3.1920 Chemnitz Solidaritätserklärung der Vollversammlung des Chemnitzer Arbeiterrats für das Ruhrproletariat (Nr. 160)

Halle Bericht des "Volksblatts“ über den Kampf der Arbeiter gegen den Kapp-Putsch in Halle (Nr. 200)

Gotha Bericht des "Gothaer Volksblatts" über die widerrechtliche Verhaftung der Gothaer Regierungsvertreter Emil Grabow und Hermann Duncker in Berlin (Nr. 241)

Rostock Bericht der sozialdemokratischen „Mecklenburgischen Volks-Zeitung“ über den Kampf der Arbeiter in Tessin und Umgebung gegen die Putschisten (Nr. 319)

Essen Das „Ruhr-Echo“ über den Entschluß der Vollversammlung der Essener Arbeiterräte, den politischen Kampf für die Erfüllung des Bielefelder Abkommens fortzusetzen und den konterrevolutionären Truppenüberfall auf das Ruhrproletariat abzuwehren (Nr. 398)

Berlin Vereinbarung zwischen der Reichsregierung und dem ADGB unmittelbar vor dem Einmarsch der Reichswehr ins Ruhrgebiet (Nr. 399)

Münster Wiedergabe des Abkommens von Münster durch die „Freiheit“ (Nr. 400)

Ende März Berlin Bericht der KPD-Bezirksorganisation Brandenburg 1920 an die Zentrale der KPD über den Kampf der Industrie- und Landarbeiter gegen den Kapp-Putsch im Bezirk (Nr. 108)

Oelsnitz (Vogtl.) Bericht aus Oelsnitz über den zunehmenden Einfluß der KPD auf die Aktionen der Arbeiter gegen die Putschisten (Nr. 159)

Magdeburg Auszug aus dem Bericht der KPD-Bezirksorganisation Magdeburg an die Zentrale der KPD über den Kampf der Arbeiter gegen den Kapp-Putsch in Magdeburg (Nr. 199)

Erfurt Bericht der KPD-Bezirksorganisation Thüringen an die Zentrale der KPD über den Generalstreik und die bewaffneten Kämpfe der Arbeiter in den westlichen Teilen Thüringens (Nr. 242)

Stuttgart Darlegung der Aufgaben der Arbeiterklasse zur Abwehr der vordringenden Konterrevolution durch die KPDBezirksorganisation Württemberg (Nr. 348) 
o. O. Appell der KPD-Bezirksorganisation Süddeutschland, zum Schutze des Proletariats im Ruhrgebiet erneut den Generalstreik zu proklamieren und den Kampf gegen die Konterrevolution mit aller Kraft fortzusetzen (Nr. 349)

Ende März/ Anfang April 1920

Anfang April 1920

1. 4.1920

Stettin Appell der Arbeiter Pommerns an die Soldaten, sich nach dem gescheiterten Putsch nicht erneut für reaktionäre Umtriebe mißbrauchen zu lassen (Nr. 320)

Lïbeck Aufforderung der Bezirkskonferenz der KPD für Mecklenburg an das Proletariat, erneut in den Generalstreik zu treten, wenn die Bourgeoisie nicht entwaffnet wird oder die Reichsregierung Truppen gegen das Ruhrproletariat einsetzt (Nr. 321)

o. O. Aufzeichnungen Ernst Puchmüllers, Dassow, über seine Verhaftung und die Ermordung des Arbeiters Hermann Litzendorf (Nr. 325)

Berlin Forderung Carl v. Ossietzkys nach Zurückdrängung der Reaktion (Nr. 109)

Falkenstein Aufforderung des Roten Vollzugsausschusses des Vogtlandes zum Eintritt in die Rote Garde (Nr. 161)

Berlin Bericht eines USPD-Funktionärs über die Kämpfe der Leipziger Arbeiter gegen den Kapp-Putsch (Nr. 162)

Halle Das „Volksblatt", Halle, über die bewaffneten Kämpfe in Halle-Trotha (Nr. 201)

Halle Auszug aus der Rede Wilhelm Koenens auf der Hallenser Konferenz am 26. März 1920 (Nr. 202)

Halle Bericht des Halleschen „Volksblatts“ über den weißen Terror in Halle (Nr. 203)

Jena Forderungen der Betriebsvertrauensleuteversammlung in Jena an die Reichsregierung, den Rachefeldzug der Konterrevolution gegen das Proletariat im Ruhrgebiet und in Westthüringen zu verhindern (Nr. 243)

Essen Anerkennung der Abkommen von Bielefeld und Münster durch den Zentralrat und die Kampfleiter der Roten Armee (Nr. 401)

Berlin Entscheidung der Reichsregierung, das Ruhrproletariat mit militärischen Machtmitteln niederzuschlagen (Nr. 402)

Berlin Zustimmung der Preußischen Staatsregierung zum Einfall der Reichswehr ins Ruhrgebiet (Nr: 403)

Chemnitz Forderung der Vollversammlung der Chemnitzer Arbeiterräte an die Regierung, Abstand von der geplanten Niederwerfung des sächsischen Proletariats zu nehmen (Nr. 163) 
Essen Aufruf des Zentralrats an die Soldaten der Roten Armee zur Einstellung des militärischen Kampfes (Nr. 404) Hagen Aufforderung der drei Arbeiterparteien des Bezirks Hagen, den Zentralrat in Essen beim geordneten Abbau der Kampffront zu unterstützen (Nr. 405)

Wiescherhöfen Oberjäger Max Zeller an das Reservelazarett I Station A in Dresden über die Greueltaten der Soldateska gegen Angehörige der Roten Armee und Krankenschwestern (Nr. 406)

2./3.4.1920 Hückeswagen, Remscheid, Cronenberg, Velbert, Langenberg, Gevelsberg, Elberfeld, Bochum Meldungen von Gemeindebehörden und Arbeiterausschüssen aus verschiedenen Orten des Ruhrgebiets an Severing über die Erfüllung des Bielefelder Abkommens durch die Arbeiter (Nr. 407)

3.4.1920 Stettin Resolutionsentwurf des Sekretärs der KPD-Bezirksorganisation Pommern, Karl Schulz, zur Solidarität mit dem vom Rachefeldzug der Militaristen bedrohten Ruhrproletariat (Nr. 322)

Münster Auszug aus der Rede Severings auf der Pressekonferenz der Reichszentrale für Heimatdienst über die Taktik der Reichsregierung gegenüber dem Ruhrproletariat (Nr. 408)

4.4.1920 Berlin Aufruf der Zentrale der KPD zum Generalstreik gegen die blutige Niederschlagung der Arbeiter im Ruhrgebiet, in Sachsen und Thüringen (Nr. 110)

5.4.1920 Barmen Anweisungen des Zentralrats der Vollzugsräte für das Industriegebiet Rheinland und Westfalen an alle Vollzugsausschüsse und Vollzugsräte Rheinland-Westfalens zum Abbau der Kampffront (Nr. 409)

6. 4.1920 Berlin Appell der Zentrale der KPD, erneut in den Generalstreik zu treten, um das Ruhrproletariat gegen den weißen Terror und die antinationale Politik der Reichsregierung zu verteidigen (Nr. 410)

Barmen Beweismaterial des Zentralrats über den Rachefeldzug der Reichswehr gegen das Ruhrproletariat (Nr. 411)

7.4.1920 Halle Zusammenfassender Bericht der Bezirksleitung der USPD für den Agitationsbezirk Halle über den Kampf der Arbeiter gegen den Kapp-Putsch im Bezirk (Nr. 204)

Neidenburg Forderungen der USPD, der SPD und der Gewerkschaften von Neidenburg an die Reichsregierung nach wirksamer Bekämpfung der Konterrevolution, Einleitung der Sozialisierung in Industrie und Landwirtschaft und Durch- 
LVIII

8. 4.1920

8./9. 4. 1920

9. 4.1920

10. 4.1920

14./15. 4. 1920
Dokumente in zeitlicher Folge

führung einer Friedenspolitik, besonders gegenüber Sowjetrußland (Nr. 280)

Berlin Aufforderung der Fraktion der Kommunistischen Partei Deutschlands (Spartakusbund) in der Generalversammlung der Betriebsräte Groß-Berlins an die linken Kräfte in der USPD, sich am Generalstreik zur Rettung der Ruhrarbeiter zu beteiligen (Nr. 111)

Chemnitz Ablehnung der politischen Konzeption von Max Hoelz durch die Bezirkskonferenz der KPD des Bezirks Erzgebirge-Vogtland (Nr. 164)

Burg Forderungen der Volksversammlungen in Burg nach Entmachtung der Konterrevolution (Nr. 205)

Berlin Die „Freiheit" über den vertragsbrüchigen Einfall der Reichswehr ins Ruhrgebiet (Nr. 412)

Berlin Protest der kommunistischen Fraktion der Betriebsräte Groß-Berlins gegen die antinationale Politik der MüllerRegierung, die den Einfall französischer Truppen in den Maingau provozierte (Nr. 112)

Berlin Carl Legien über die Haltung der freien Gewerkschaften im Kapp-Putsch (Nr. 113)

Barmen Erklärung des Zentralrats über seine Bemühungen zum organisierten Abbruch des Ruhrkampfes (Nr. 413)

o. O. Aufruf der Kommunistischen Partei in Sachsen (Spartakusbund) an die deutsche Arbeiterklasse, durch einen erneuten Generalstreik eine Wiederholung des weißen Terrors vom Ruhrgebiet in Sachsen zu verhindern (Nr.165)

Rostock Bericht der „Volkswacht", Rostock, über die Konferenz der Aktionsausschüsse Mecklenburgs in Güstrow (Nr. 323)

Elberfeld Aussage eines Kompanieführers der Roten Armee aus Mülheim über Brutalitäten der Reichswehrtruppen (Nr. 414)

Rostock Bericht der Rostocker „Volkswacht" über die Verhandlungen zwischen Vertretern des Aktionsausschusses Rostock und der Schweriner Regierung am 6. April 1920 zwecks Einreihung der Rostocker Arbeiterwehr in die staatliche Sicherheitspolizei (Nr. 324)

Berlin Niederschrift über die Besprechung von Ruhrindustriellen mit dem Reichspräsidenten am 10. April vormittags über die vollständige Niederschlagung der Arbeiter im gesamten westlichen Industriegebiet (Nr. 415)

Berlin Resolution der KPD zum Kapp-Putsch (Nr. 114) 
15. 4.1920

16. 4.1920

22. 4.1920

25. 4.1920

26. 4.1920

28. 4.1920

30. 4.1920

April 1920

April/Mai 1920

Mai 1920

12. 5.1920

18. 6.1920
Jena Bericht der „Neuen Zeitung“, Jena, über die Konferenz der Aktionsausschüsse des Landes Sachsen-Weimar-Eisenach am 8. April 1920 in Weimar (Nr. 244)

Karow Aufzeichnung von Zeugenaussagen über die brutale Mißhandlung und Ermordung des Funktionärs des Landarbeiterverbandes Franz Slomski am 18. März 1920 in Dorf Mecklenburg (Nr. 326)

Meerane Protest einer öffentlichen Volksversammlung in Meerane gegen die Verhängung des Belagerungszustandes (Nr. 166)

Erfurt Appell der Bezirksleitung der KPD von Thüringen an die Ortsgruppen zur finanziellen Unterstützung der Opfer des Kapp-Putsches (Nr. 245)

Berlin „Die Rote Fahne“ zum Problem der Arbeiterregierung im Zusammenhang mit den Reichstagswahlen (Nr. 115) Bochum Auszug aus dem Bericht des Polizeidirektors von Bochum an den Regierungspräsidenten in Arnsberg über den Kampf der Bochumer Arbeiter gegen den Kapp-Putsch (Nr. 416)

Niendorf Bericht des Gendarmerie-Oberkommissars Göldenitz mit Zeugenaussagen über die Ermordung der Landarbeiter Wittke und Steinfurth (Nr. 327)

Erfurt Bericht des „Kommunist“, Erfurt, über das Wüten der Konterrevolution in Schallenburg und Sömmerda (Nr. 246)

Berlin Siegfried Aufhäuser über die Haltung der Afa während des Kapp-Putsches (Nr. 116)

Wesel Vorgedrucktes Urteilsformular mit Urteilsgründen des außerordentlichen Kriegsgerichts Wesel gegen Angehörige der Roten Armee (Nr. 417)

o. O. Auszug aus Aufzeichnungen von Urteilen der außerordentlichen Kriegsgerichte Wesel und Essen gegen Ruhrarbeiter (Nr. 418)

o. O. Auszug aus Aufzeichnungen von Urteilen des außerordentlichen Kriegsgerichts Buer gegen Krankenschwestern der Roten Armee (Nr. 419)

Münster Anweisung des Oberpräsidenten und Regierungskommissars der Provinz Westfalen an das Kommando der Sicherheitspolizei in Münster über die Entwaffnungsaktion im Gebiet südlich der Ruhr (Nr. 420)

Marburg Zeugenaussage des Offizierstellvertreters Dahlheim vor dem Kriegsgericht der ehemaligen 22. Division gegen die Mörder der 15 Arbeiter aus Bad Thal (Nr. 247) 
24.9.1920 München Hauptmann Karl Mayr an Kapp über die Konterrevolution in Bayern, die sich auf den Faschismus orientiert (Nr. 117)

Oktober 1920 o. O. Gemeinsamer Aufruf des EKKI, der Zentrale der KPD und des Zentralkomitees der USPD (Linke) vom Oktober 1920 zur Bildung einer Vereinigten Kommunistischen Partei Deutschlands (Nr. 120)

3. 3. 1921 Berlin Die „Freiheit“ über den Prozeß gegen die Mörder aus dem Freikorps Aulock in Breslau (Nr. 281)

21.12.1921 Leipzig Urteil des Reichsgerichts im Hochverratsprozeß gegen die Putschisten Jagow, Wangenheim und Schiele (Nr. 118)

22.12.1921 Berlin „Die Rote Fahne“ über die Klassenjustiz im JagowProzeß (Nr. 119)

Juli 1924 Berlin Antrag der kommunistischen Reichstagsfraktion auf Entschädigung für die von der Soldateska bestialisch mißhandelte Arbeiterin Maria Lippert und für alle Opfer des KappPutsches (Nr. 421)

O. D. o. O. Auszug aus der Denkschrift Kapps über die Vorbereitung und Auslösung des Putsches (Nr. 33) 